\title{
Low-frequency conductivity of a two-dimensional Wigner solid coupled to surface excitations of liquid helium
}

\author{
Yu.P. Monarkha \\ B. Verkin Institute for Low Temperature Physics and Engineering \\ of the National Academy of Sciences of Ukraine, 47 Lenin Ave., Kharkov 61103, Ukraine
}

Received March 26, 2003

\begin{abstract}
The line-shape and broadening of coupled phonon-ripplon resonances of Wigner-solid conductivity are studied using the memory function formalism. The analytic properties of the memory function permit coordinating the approximations of the secular equation for the coupled phonon-ripplon modes and the line-broadening of these resonances. Special attention is paid to the description of the strong-coupling regime realized for surface electrons on superfluid helium. For this case it is shown that the line-broadening is much smaller than and the line-shape is different from those found previously using the weak-coupling theory. Different theoretical approaches are compared with available experimental data.
\end{abstract}

PACS: $67.40 . \mathrm{Db}, \mathbf{7 3 . 2 0 .}-\mathbf{r}$

\section{Introduction}

A Wigner solid (WS) is usually associated with the insulator state of an electron system. In such highly correlated state the electrons are assumed to be pinned by static media defects or boundary-shape distortions. A remarkable exception is the two-dimensional (2D) WS formed on the free surface of liquid helium. In this case there are no static media defects and the edges of the electron sheet are formed by the external electric fields of the guard electrodes. In the presence of an ac driving electric field such WS can move along the helium surface, interacting with media excitations.

At typical temperatures $T \lesssim 0.5 \mathrm{~K}$, which can be associated with the WS state for areal electron densities $n_{s} \sim 10^{8}-10^{9} \mathrm{~cm}^{-2}$, electrons interact predominantly with capillary wave quanta (ripplons). Because each electron is localized near its lattice site, the electron-ripplon interaction induces a sublattice of surface dimples [1]: a static surface displacement $\xi(\mathbf{r})=$ $=\sum_{\mathbf{g}} \xi_{\mathbf{g}}^{(0)} \exp (i \mathbf{g r})$, where $\mathbf{g}$ is the reciprocal lattice vec-

tor. Thus, the electrons become self-pinned to the surface dimples, which can move and follow slow electrons. In other words phonons of the WS formed on the surface of liquid helium are strongly coupled to the media (surface) excitations. Therefore, the low- frequency excitation spectrum of the WS interacting with ripplons differs significantly from that established for a 2D electron solid without interactions [2].

The observation of the coupled phonon-ripplon modes with $\omega<\omega_{g_{1}}=\sqrt{\alpha / \rho} g_{1}^{3 / 2}$ (here $\alpha$ and $\rho$ are the surface tension and mass density of liquid helium, respectively, and $\mathbf{g}_{1}$ is the smallest reciprocal lattice vector) and electron-ripplon resonances at higher frequencies $\omega=\omega_{g}$, with larger $g$, have served as unique proof of the ordered state of the electron system [3]. Considerable research has been performed on the theory describing these coupled modes and the positions of the electron-ripplon resonances [4-6]. At the same time, there is a lack of theoretical research on the line-broadening and line-shape of the electron-ripplon resonances in the strong-coupling regime which can be used for the analysis of the large body of data that is now available [7-9].

Another important issue related to the electron self-pinning to the sublattice of surface dimples is a remarkable nonlinear conductivity of the WS reported for surface electrons on liquid helium [10,11]. Possible explanations of this phenomenon also involve the concept of strong coupling of the Wigner lattice to surface excitations of liquid helium. Therefore, an accurate description of the conductivity of the electron crystal interacting strongly with media excitations is very important for understanding the unusual proper- 
ties of «solid» currents observed for surface electrons on liquid helium.

The real part of the conductivity of a highly correlated electron system in a nonuniform electric field $\mathbf{E}_{\mathbf{k}}$ with a small excitation wave-vector $\mathbf{k}$ directed along the electric field can be quite generally written in the form [12]

$\operatorname{Re} \sigma_{k}(\omega)=\frac{e^{2} n_{s}}{m} \frac{v(\omega)}{\left[\omega+\omega(\omega)-\Omega_{l}^{2}(k) / \omega\right]^{2}+v^{2}(\omega)}$,

where $m_{e}$ is the free electron mass and $\Omega_{l}(k) \equiv \Omega_{l, k}$ is the plasmon spectrum or the spectrum of longitudinal phonons of the WS established for the flat surface. The auxiliary functions $v(\omega)$ and $w(\omega)$ make up the conductivity relaxation kernel $M(\omega)=w(\omega)+$ $+i v(\omega)$, usually called the memory function. The imaginary part of the relaxation kernel $v(\omega)$ can be called the effective collision frequency because it determines the kinetic friction acting on the electron system because of the interaction with scatterers.

The real part $w(\omega)$ describes the non-dissipative force acting on electrons because of the induced media polarization cloud. This force is proportional to the average electron displacement $\mathbf{u}$ induced by an ac driving electric field. If $w(\omega)$ can be disregarded, Eq. (1) describes the power absorption because of the resonant excitation of 2D plasmons. For the WS state of the electron system realized on liquid helium, $w(\omega)$ determines the secular equation for the coupled phononripplon modes:

$$
\omega+w(\omega)-\Omega_{p, k}^{2} / \omega=0,
$$

where the subscript $p$ denotes the polarization: $p=(l, t)$. At frequencies satisfying this equation with $p=l$, the power absorption proportional to $\operatorname{Re}\left[\sigma_{k}(\omega)\right]$ increases in a resonance manner. In the theory of the coupled phonon-ripplon modes the function $\omega(\omega)$ has singularities at typical ripplon frequencies $\left(\omega=\omega_{g}\right)[2,4]$ :

$$
\omega \approx(\omega) \propto \frac{\omega^{2}}{\omega_{g}^{2}-\omega^{2}} .
$$

Therefore $\omega_{g_{1}}$ is the upper bound for the slow coupled phonon-ripplon modes $\Omega_{p, k}^{(s)}<\omega_{g_{1}}$, as shown in Fig. 1.

This figure shows only a very small part of the first Brillouin zone with $k=|\mathbf{k}|<<g$. The lowest dotted horizontal line indicates the ripplon spectrum at much larger wave-numbers $q=\left|\mathbf{g}_{1} \pm \mathbf{k}\right| \simeq g_{1}$ represented in the first Brillouin zone. There are also coupled modes below each of the higher ripplon frequen-

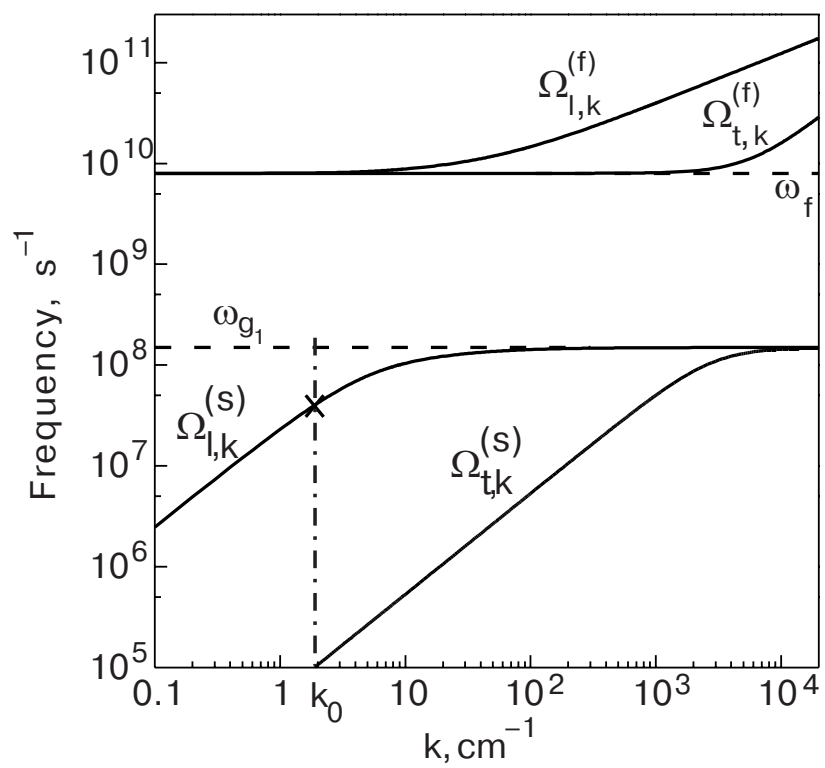

Fig. 1. View of the spectrum of the WS phonon modes coupled to media vibrations (ripplons) for $n_{S}=9.5 \cdot 10^{8} \mathrm{~cm}^{-2}$.

cies $\omega_{g}>\omega_{g_{1}}$ which are not shown in Fig. 1. The fast modes $\Omega_{p, k}^{(f)}$ are determined as the phonon modes affected by the field of static surface dimples: $\Omega_{p, k}^{(f)}=\sqrt{\omega_{f}^{2}+\Omega_{p, k}^{2}}$, where $\omega_{f}$ is the frequency of single electron oscillations in a dimple.

In the strong coupling regime $\omega_{f}>\omega_{g_{1}}$ usually realized for the surface electrons on liquid helium, the secular equation and the positions of the resonances are well described by the self-consistent approach proposed in Refs. 5, 6. The characteristic frequencies $\omega_{g_{1}}$ and $\omega_{f}$ introduce a natural separation of electron displacements $\mathbf{u}_{1}$ from the lattice sites into the slow and fast parts $\left(\mathbf{u}_{1}=\mathbf{u}_{s, 1}+\mathbf{u}_{f, 1}\right)$, as follows from Fig. 1 . Therefore, the equation of motion for the slow modes $\Omega_{p, k}^{(s)}$ (or equivalently the interaction Hamiltonian) can be averaged over the fast vibrations, which introduces the high-frequency Debye-Waller factor (DWF) exp $\left(-q^{2}\left\langle u_{f}^{2}\right\rangle / 4\right)$. Owing to the limiting frequency $\omega_{f}$, the mean-square displacement of the fast mode $\left\langle u_{f}^{2}\right\rangle$ does not exhibit the logarithmic dependence on the linear dimensions of the sample that is typical for $2 \mathrm{D}$ solids. The quantities $\left\langle u_{f}^{2}\right\rangle$ and $\omega_{f}$ are found by a self-consistent procedure. Good agreement between the theory and experiment has been reported for the slow modes in the temperature range $0.2 \mathrm{~K} \leq T \leq 0.6 \mathrm{~K}[8]$.

The ultra-low temperature measurements of the high-frequency DWF conducted in Ref. 13 also agree well with the self-consistent theory in the entire temperature range. This means that the real part of the conductivity relaxation kernel $w(\omega)$ has been well es- 
tablished. In contrast, the imaginary part $v(\omega)$ has been analyzed only for the weak coupling regime [14] or high-frequency conditions $\omega \sim \omega_{f} \gg \omega_{g}$ [15]. In this paper we use the memory function formulation of the electron conductivity [16] to describe the electron-ripplon resonances and the dc mobility of the WS in the regime of strong coupling with media excitations.

It should be emphasized that in the memory function approach $v(\omega)$ and $w(\omega)$ are not entirely independent because they are the imaginary and real parts of the memory function which possesses certain analytical properties [16]. They must obey the KramersKronig relations. Both parts can be expressed in terms of the electron dynamical structure factor (DSF) $S(\mathbf{q}, \omega)$. Therefore, the approximations for $S(\mathbf{q}, \omega)$ and $v(\omega)$ must be consistent with $w(\omega)$ and with the well-established secular equation for the coupled phonon-ripplon modes. In this paper we show that some important conductivity results from the weak-coupling theory, formally extended to the strong-coupling regime, do not satisfy this consistency requirement and overestimate $v(\omega)$ significantly.

We have analyzed the properties of $S(\mathbf{q}, \omega)$ and $v(\omega)$ in the strong-coupling regime and found that the discrepancy primarily originates from the fact that a significant part of the electron-ripplon interaction $\mathrm{Ha}-$ miltonian

$$
\begin{aligned}
& H_{\text {int }}=\frac{1}{\sqrt{S_{A}}} \sum_{\mathbf{q}} V_{q} \xi_{\mathbf{q}} \sum_{\mathbf{l}} \mathrm{e}^{i \mathbf{q} \mathbf{R}_{\mathbf{l}}+i \mathbf{q}\left(\mathbf{u}_{s, 1}+\mathbf{u}_{f, 1}\right)}= \\
& =\frac{1}{\sqrt{S_{A}}} \sum_{\mathbf{q}} V_{q} \xi_{\mathbf{q}} \sum_{\mathbf{1}}\left[1+i \mathbf{q} \mathbf{u}_{s, 1}+\ldots\right] \mathrm{e}^{i \mathbf{q} \mathbf{R}_{\mathbf{1}}+i \mathbf{q} \mathbf{u}_{f, 1}}
\end{aligned}
$$

(here $\mathbf{R}_{1}$ is the lattice-site vector, $V_{q}$ is the electron-ripplon coupling, and $S_{A}$ is the surface area) is included in the Hamiltonian of the new slow modes causing the strong renormalization of the WS phonon spectrum $\Omega_{p, k}^{(s)}$. This concerns the second term of the expansion enclosed in the square brackets $[\ldots]$, and even the next term $\left(i \mathbf{q u}_{s, \mathbf{1}}\right)^{2} / 2$ when $\xi_{\mathbf{q}} \simeq \xi_{\mathbf{q}}^{(0)}$. These terms play an essential role in the secular equation for the slow modes.

In the weak-coupling theory the interaction proportional to $V_{q} \xi_{\mathbf{q}} i \mathbf{q u} \mathbf{u}_{s, 1}$ describes scattering events involving one slow phonon $\left(\Omega_{p, k}^{(s)}\right)$. Therefore, any approximation for the electron DSF in the strong-coupling regime should exclude these scattering events in order to avoid double counting the contribution due to the term $i \mathbf{q u}_{s, \mathbf{1}}$. This reduces significantly the effective collision frequency $v(\omega)$ as compared to the result found by formal extension of the weak coupling theory, and affects the line-shape of the electron-ripplon resonances.
The discussion above is not applicable to the fast phonon modes because they represent electron oscillations in the presence of the field due to static dimples $\xi_{g}^{(0)}$. Anyway, at low frequencies one-phonon processes involving $\Omega_{p, k}^{(f)}$ and $\omega_{g}$ are forbidden because $\omega_{f}>>\omega_{g}$. In this paper we shall also analyze multi-phonon processes for slow and fast modes. This analysis can be used to describe the conductivity of the WS in the strong-coupling regime. The results are compared with available experimental data.

\section{Basic relations}

Götze and Wölfle [16] proposed the following approximation for the conductivity relaxation kernel

$$
M(\omega)=\frac{1}{m_{e} \omega N_{e}}\left[G_{F_{x} F_{x}}^{(R)}(0)-G_{F_{x} F_{x}}^{(R)}(\omega)\right],
$$

where $N_{e}=n_{S} S_{A}$ is the number of electrons, $\mathbf{F}=-\sum \partial H_{\mathrm{int}} / \partial \mathbf{r}_{e}$ is the force owing to the interaction with $\stackrel{e}{s}$ catterers, $G_{A B}^{(R)}(\omega)$ is the conventional retarded Green's function for two operators $A$ and $B$

$$
G_{A B}^{(R)}(\omega) \equiv\langle\langle A ; B\rangle\rangle_{\omega}=-\frac{i}{\hbar} \int_{0}^{\infty} \mathrm{e}^{i \omega t}\langle[A(t), B(0)]\rangle d t,
$$

and \langle\rangle means averaging over equilibrium distributions. Even though for the main interactions Eqs. (1) and (4) reproduce the well-known results of the kinetic equation method in the entire frequency range, in general Eq. (4) is a high-frequency approximation $(\omega>>v)$. Therefore at low frequencies, for certain coupling potentials $V_{q}$, it may give a wrong numerical proportionality factor of the order of 2 . A remarkable exception is a highly correlated electron liquid, where the electron-electron collision rate $v_{e e}>>v$. In this case the approximation Eq. (4) is proven to be valid at all relevant frequencies [12]. The Wigner solid certainly satisfies this requirement.

The real and imaginary parts of the memory function are related with one another because of the well-known representation

$$
G_{F_{x} F_{x}}^{(R)}(\omega)=\int_{-\infty}^{\infty}\left\langle F_{x}(t) F_{x}(0)\right\rangle_{\omega^{\prime}} \frac{\left(1-\mathrm{e}^{-\hbar \omega^{\prime} / T}\right)}{\omega-\omega^{\prime}+i 0} \frac{d \omega^{\prime}}{2 \pi \hbar},
$$

where \langle\rangle$_{\omega}$ is the Fourier transform of the correlation function. For the interaction Hamiltonian $H_{\text {int }}$ given in Eq. (3), the above introduced force operator $\mathbf{F}$ can be written in the form

$$
\mathbf{F}=-\frac{i}{\sqrt{S_{A}}} \sum_{\mathbf{q}} \mathbf{q} V_{q} \xi_{\mathbf{q}} n_{-\mathbf{q}}
$$


where $n_{-\mathbf{q}}=\sum_{1} \exp \left(i \mathbf{q r}_{\mathbf{l}}\right)$ is the electron density fluctuation operator. Straightforward evaluation of the force-force correlation function yields

$$
\begin{gathered}
\left\langle F_{x}(t) F_{x}(0)\right\rangle_{\omega}=n_{s} \sum_{\mathbf{q}} q_{x}^{2} V_{q}^{2} Q_{q}^{2} \times \\
\times\left[\left(N_{q}+1\right) S\left(\mathbf{q}, \omega-\omega_{q}\right)+N_{q} S\left(\mathbf{q}, \omega+\omega_{q}\right)\right],
\end{gathered}
$$

where $Q_{q}=\sqrt{\hbar q / 2 \rho \omega_{q}}$, and $N_{q}$ is the equilibrium ripplon distribution function. The electron DSF $S(\mathbf{q}, \omega)$ is defined in the usual way

$$
S(\mathbf{q}, \omega)=N_{e}^{-1} \int_{-\infty}^{\infty} \mathrm{e}^{i \omega t}\left\langle n_{\mathbf{q}}(t) n_{-\mathbf{q}}(0)\right\rangle d t .
$$

Thus, Eqs. (4), (6) and (8) make it possible to express the real and imaginary parts of the conductivity relaxation kernel in terms of the electron DSF.

Under the usual experimental conditions $N_{q} \simeq$ $\simeq T / \hbar \omega_{q}$. Therefore the effective collision frequency can be written in the very simple form

$$
\begin{gathered}
v(\omega)=\frac{\left(1-\mathrm{e}^{-\hbar \omega / T}\right) T}{8 \alpha m_{e} S_{A} \hbar \omega} \sum_{\mathbf{q}} V_{q}^{2}\left[S\left(\mathbf{q}, \omega-\omega_{q}\right)+\right. \\
+S\left(\mathbf{q}, \omega+\omega_{q}\right) .
\end{gathered}
$$

At the same time, the real part of the conductivity relaxation kernel $w(\omega)$ contains additional integration over the frequency argument $\omega^{\prime}$ with the factor $\left(\omega-\omega^{\prime}\right)^{-1}$ according to Eq. (6).

Let us ignore, at first, the separation of electron displacements into slow and fast modes and consider possible approaches to evaluating the electron DSF. The conventional procedure is to rewrite Eq. (9) in the following form employing the Bloch identity and the algebra of noncommuting operators:

$$
S(\mathbf{q}, \omega)=\sum_{1} \mathrm{e}^{-i \mathbf{q} \mathbf{R}_{\mathbf{1}}} \int_{-\infty}^{\infty} \mathrm{e}^{i \omega t-h_{q}(1, t)} d t,
$$

where

$$
\begin{gathered}
h_{q}(\mathbf{l}, t)=2 q^{2}[W(0,0)-W(\mathbf{l}, t)], \\
W(\mathbf{l}, t)=\frac{\hbar}{4 N_{e} m_{e}} \sum_{p, \mathbf{k}} \frac{\left|E_{p, \mathbf{k}}^{(q)}\right|^{2}}{\Omega_{p, k}} \times \\
\times\left[\left(n_{p, k}+1\right) \mathrm{e}^{i\left(\mathbf{k R}_{\mathbf{1}}-\Omega_{p, k} t\right)}+n_{p, k} \mathrm{e}^{-i\left(\mathbf{k R}_{\mathbf{1}}-\Omega_{p, k} t\right)}\right],
\end{gathered}
$$

$n_{p, k}$ is the WS phonon distribution function and $E_{p, \mathbf{k}}^{(q)}$ is the projection of the phonon polarization vector $\mathbf{E}_{p, \mathbf{k}}$ onto the direction of $\mathbf{q}$.

At high temperatures, the exponent $h_{q}(1, t)$ is large and it is reasonable to use the short-time approximation, expanding $h_{q}(1, t)$ in powers of $\Omega_{p, k} t$ up to second-order terms near its minimum. In this limit the DSF of the electron solid coincides with the DSF of a nondegenerate electron gas. This conclusion has an analogy in the theory of neutron scattering by crystals, where the solid target can be approximated by a nuclear gas.

At low temperatures in ordinary solids the conventional approach is to expand the exponential in Eq. (11) in powers of $2 q^{2} W(\mathbf{1}, t)$. This gives zero(elastic), one-, two- and other multi-phonon terms. This is called the phonon expansion. For example, the first two terms of this expansion can be written as

$$
S^{(\mathrm{elas})}(\mathbf{q}, \omega)=2 \pi \mathrm{e}^{-2 W_{q}} \delta(\omega) N_{e} \sum_{\mathbf{g}} \delta_{\mathbf{q}, \mathbf{g}},
$$

$$
\begin{gathered}
S^{(1-\mathrm{ph})}=\frac{\pi \hbar q^{2}}{2 m_{e}|\omega|}\left\{\left[n_{\mathrm{B}}(\hbar|\omega|)+1\right] \sum_{\mathbf{k}}\left|\mathbf{E}_{t, \mathbf{k}}\right|^{2} \delta\left(\omega-\Omega_{t, k}\right)+\right. \\
\left.+n_{B}(\hbar|\omega|) \sum_{\mathbf{k}}\left|\mathbf{E}_{t, \mathbf{k}}\right|^{2} \delta\left(\omega+\Omega_{t, k}\right)\right\} \mathrm{e}^{-2 W_{q}} \sum_{\mathbf{g}} \delta_{\mathbf{q}, \mathbf{g}+\mathbf{k}},
\end{gathered}
$$

where $n_{B}(\hbar \omega)$ is the Bose distribution function, $W_{q}=q^{2} W(0,0)$, and we have taken into account the fact that the main contribution to $S^{(1-\mathrm{ph})}$ comes from transverse phonons. In conventional systems $\left|\mathbf{E}_{t, \mathbf{k}}\right|^{2}=1$. We shall retain this factor in Eq. (14), so that it is applicable to the slow phonon modes of the strongly coupled system, where $\left|\mathbf{E}_{t, \mathbf{k}}\right|^{2} \neq 1$. The factor $\delta_{\mathbf{q}, \mathbf{g}+\mathbf{k}}$ taken out of the brackets $\{.$.$\} indicates that in$ one-phonon processes a WS phonon interacts with a ripplon of the wave-vector $\mathbf{q}=\mathbf{g} \pm \mathbf{k}$. In most cases, typical phonon wave-vectors $k<<g$ and $\delta_{\mathbf{q}, \mathbf{g} \pm \mathbf{k}}$ can be replaced by $\delta_{\mathbf{q , g}}$.

In the elastic approximation $\left[S(\mathbf{q}, \omega) \simeq S^{(\mathrm{elas})}(\mathbf{q}, \omega)\right]$ the conductivity relaxation kernel can be evaluated employing Eqs. (6) and (13):

$$
\begin{gathered}
\omega(\omega)=\frac{n_{s}}{2 \omega m_{e} \alpha} \sum_{\mathrm{g}} V_{g}^{2} \mathrm{e}^{-2 W_{g}} \frac{\omega^{2}}{\omega_{g}^{2}-\omega^{2}}, \\
v(\omega)=\frac{\pi n_{s}\left(1-\mathrm{e}^{-\hbar \omega / T}\right) T}{4 \alpha m_{e} \hbar \omega} \sum_{\mathrm{g}} V_{g}^{2} \mathrm{e}^{-2 W_{g}} \delta\left(\omega-\omega_{g}\right) .
\end{gathered}
$$

The real part $w(\omega)$ of Eq. (15) gives the proper secular equation for the slow coupled phonon modes, if 
$2 W_{g} \rightarrow g^{2}\left\langle u_{f}^{2}\right\rangle / 2$. It is important that the effective collision frequency of electrons forming the 2D Wigner solid has a resonance structure itself, which agrees with the hydrodynamic model $[17,18]$. Equations (15) and (16) with the self-consistent DWF can be also found directly by evaluating the force acting on the Wigner lattice induced by slow uniform displacements $\left(\mathbf{u}_{\mathrm{s}}(t) \propto \mathrm{e}^{-i \omega t}\right)$,

$$
\frac{\mathbf{F}(t)}{N_{e}}=m_{e} \omega[\omega(\omega)+i v(\omega)] \mathbf{u}_{\mathrm{s}}(t),
$$

when the surface displacements $\xi_{\mathbf{q}}(t)$ are described by the usual capillary wave equations. In the latter treatment the averaging over the fast modes is an accurate procedure which results in the appearance of the self-consistent DWF exp $\left(-g^{2}\left\langle u_{f}^{2}\right\rangle / 2\right)$.

For 2D solids the conventional low-temperature phonon expansion is problematic because $W(0,0)=$ $=\left\langle u^{2}\right\rangle / 4$ diverges as the linear dimension $L \rightarrow \infty$. The correct treatment has been proposed in Refs. 19, 20. It represents $h_{q}$ as a logarithmic function of $R_{1}$. For surface electrons on liquid helium a similar approximation was used in Refs. 14, 21:

$$
h_{q}(\mathbf{l}, t) \approx \alpha_{q}(T) \ln \left(\omega_{m} \sqrt{t^{2}+b R_{1}^{2} / c_{t}^{2}}\right)+q^{2}\left\langle u_{0}^{2}\right\rangle / 2,
$$

where

$$
\alpha_{q}(T)=\frac{q^{2} T}{4 \pi m_{e} c_{t}^{2} n_{s}},
$$

$\left\langle u_{0}^{2}\right\rangle$ is the zero-point mean-square displacement, $b$ is a number of the order of $1, \omega_{m}=\min \left(T / \hbar, c_{t} k_{m}\right)$, and $k_{m}=\sqrt{4 \pi n_{s}}$. At low temperatures $\omega_{m}=T / \hbar$, because the logarithmic increase of $\left\langle u^{2}\right\rangle$ at large wavenumbers is cut off by the Bose distribution function.

According to Refs. 14, 21 the time dependence of the approximation for $h_{q}(\mathbf{l}, t)$ given above smears the $\delta$-shaped spikes in the frictional force and the effective collision frequency in Eq. (16). The final results can be expressed as a long-time approximation for the effective WS dynamical structure factor

$$
S(\mathbf{q}, \omega) \simeq \frac{\pi \zeta_{q} N_{e}}{\omega_{m}^{\alpha_{q}}|\omega|^{1-\alpha_{q}}} \mathrm{e}^{-q^{2}\left\langle u_{0}^{2}\right\rangle / 2} \sum_{\mathbf{g}} \delta_{\mathbf{q}, \mathbf{g}},
$$

where

$$
\zeta_{q}=\frac{2}{\pi} \sin \left(\pi \alpha_{q} / 2\right) \Gamma\left(1-\alpha_{q}\right),
$$

and $\Gamma(x)$ is the gamma function. Then the WS collision frequency can be written in the form

$$
\begin{aligned}
& v(\omega)=\frac{\pi n_{s}}{8 \alpha m_{e} \omega_{m}} \sum_{\mathrm{g}} \zeta_{g}\left|V_{g}\right|^{2} \mathrm{e}^{-g^{2}\left\langle u_{0}^{2}\right\rangle / 2} \times \\
& \times\left[\left|\frac{\omega_{m}}{\omega-\omega_{g}}\right|^{1-\alpha_{g}(T)}+\left|\frac{\omega_{m}}{\omega+\omega_{g}}\right|^{1-\alpha_{g}(T)}\right] .
\end{aligned}
$$

The conductivity obtained in Ref. 14 can be found employing this expression and the general relation Eq. (1). The nonresonant term in the square brackets in Eq. (22) was previously disregarded. If $\alpha_{g}<<1$, the dimensionless factor $\zeta_{g} \rightarrow \alpha_{g}(T) \propto T$. The important points are that Eq. (22) does not transform into Eq. (16) at $T \rightarrow 0$ and the resonance structure of the effective collision frequency has unusual tails: the frictional force increases as a power law of the reciprocal detuning $\left|\omega-\omega_{g}\right|^{-1}$ with a temperature-dependent exponent $1-\alpha_{g}(T)$. The line-shape of the electron-ripplon resonances which follows from the conductivity equation given in Ref. 14 has a tail that decays much more weakly than in the usual Lorentzian form. These results have also been used to describe of the Bragg-Cherenkov scattering and the nonlinear conductivity of the WS [21].

\section{Weak-coupling treatment}

The approximation of Eq. (18) and the result of Eq. (22) were found neglecting the changes induced in the WS phonon spectrum by the phonon-ripplon interaction. This means that strictly speaking they correspond to the weak coupling regime. The important question is why $S(\mathbf{q}, \omega)$ and $v(\omega)$ do not transform into the result given by the elastic approximation if $T \rightarrow 0$. A related important point is that the approximation of Eq. (20) for the electron DSF is not consistent with the secular equation for the coupled phonon-ripplon modes because it cannot reproduce Eq. (15) for the real part of the conductivity relaxation kernel.

The answer can be anticipated, if we note that $v$ in Eq. (22) is proportional to $\alpha_{g}(T)$ when $\alpha_{g}(T)<<1$. It is clear that it relates somehow to the one-phonon term [Eq. (14)] of the conventional low-temperature expansion. Indeed, the main contribution to the sums $\sum_{\mathrm{k}}$ containing the delta-functions is due to quite large wavenumbers $k_{t}=\omega / c_{t}$ (much larger than $k_{0} \sim 1 / L$ ). Then, for phonons with $k \gtrsim k_{t}$ only, we can separate $W(0,0)$ and $W(\mathbf{l}, t)$ in $h_{q}(\mathbf{l}, t)$ and expand the exponential function in Eq. (11) in powers of $2 q^{2} W(\mathbf{1}, t)$, assuming that the rest of $h_{q}(\mathbf{l}, t)$ is small at low enough temperatures. Comparing with the result given in Eq. (22) for arbitrary $\alpha_{g}(T)<1$ will show if the lat- 
ter assumption is true. For example, if it is not true and the rest of $h_{q}(1, t)$ is important at $\alpha_{g}(T)<<1$, we will arrive at a different asymptotic behavior as $T \rightarrow 0$.

Assuming that $\Omega_{t, k}=c_{t} k$ is not affected by ripplons, $\left|\mathbf{E}_{t, \mathbf{k}}\right|^{2}=1$, and $\hbar|\omega|<<T$ Eq. (14) can be transformed into the form

$$
\begin{gathered}
S^{(1-\mathrm{ph})}(\mathbf{q}, \omega) \simeq \frac{\hbar q^{2} S_{A}}{4 m_{e} c_{t}^{2}} \mathrm{e}^{-q^{2}\left\langle u_{f}^{2}\right\rangle / 2} \frac{T}{\hbar|\omega|} \sum_{\mathbf{g}} \delta_{\mathbf{q}, \mathbf{g}}= \\
=\frac{\pi \alpha_{q}(T)}{|\omega|} \mathrm{e}^{-q^{2}\left\langle u_{f}^{2}\right\rangle / 2} N_{e} \sum_{\mathbf{g}} \delta_{\mathbf{q}, \mathbf{g}},
\end{gathered}
$$

where the factor $T / \hbar|\omega|$ originates from the phonon distribution function $n_{B}(\hbar|\omega|) \simeq T / \hbar|\omega|$. This factor is responsible for the unusual resonance structure of the effective collision frequency which can be evaluated employing the general relation Eq. (10)

$$
\begin{aligned}
v^{(1-\mathrm{ph})}(\omega) & =\frac{\pi n_{s}}{8 \alpha m_{e}} \sum_{\mathrm{g}} \alpha_{g}(T) V_{g}^{2} \mathrm{e}^{-g^{2}\left\langle u_{f}^{2}\right\rangle / 2} \times \\
& \times\left(\frac{1}{\left|\omega-\omega_{g}\right|}+\frac{1}{\left|\omega+\omega_{g}\right|}\right) .
\end{aligned}
$$

The resonance structure of the effective collision frequency as $\omega \rightarrow \omega_{g}$ is described by the first term in parentheses. Comparing Eq. (24) with the previously found result [Eq. (22)] shows that these equations are equivalent in the limiting case $\alpha_{g}(T)<<1$. The comparison also shows the effect of long-wavelength fluctuations in the 2D electron crystal on the conventional one-phonon term. The long-wavelength fluctuations just change the exponent of the resonant term $\left(\left|\omega-\omega_{g}\right|^{-1} \rightarrow\left|\omega-\omega_{g}\right|^{-1+\alpha_{g}}\right)$ and restrict the proportionality factor $\alpha_{g}(T)$ when it becomes of the order of 1 . Both these effects just reduce the result of the conventional one-phonon term [Eq. (24)]. Therefore, we see that in the limiting case $\alpha_{g}(T)<<1$ Eq. (22) transforms into the one-phonon term of the conventional phonon expansion rather then into the elastic term.

Thus the approximations for $S(\mathbf{q}, \omega)$ and $v(\omega)$ given in Eqs. (20) and (22) at low temperatures should not be treated as substitutes for the zero-temperature forms of Eqs. (13) and (16), but rather as the forms that should be added to them. This can be verified in terms of the approximation given in Eq. (18) by expanding formally the proper time integral in powers of the small parameter $\alpha_{q}$. In order to guarantee the convergence of the time integral we can introduce an additional infinitesimal parameter and set it to zero in the final result. This gives the following transformation of the conventional $\delta$-spikes:

$$
\delta(\omega) \rightarrow \delta(\omega)+\frac{\alpha_{q}(T)}{2|\omega|},
$$

if $\alpha_{q}<1$. This agrees with the elastic and onephonon terms in the conventional phonon expansion. The important point is that in this case we regain the proper form for the real part of the memory function and the secular equation for the coupled modes, at least for $\alpha_{q}<<1$.

Comparing Eqs. (22) and (24) one can conclude that the long-wavelength fluctuations can be disregarded when $\alpha_{g}(T)<<1$. For the first reciprocal lattice vector, at the WS melting temperature the parameter $\alpha_{g}\left(T_{m}\right)=1 / 3$. It increases rapidly with $g$ because $\alpha_{g}(T) \propto g^{2}$. This affects electron-ripplon resonances with higher frequencies $\omega_{g}$. For steady motion of the WS with a constant velocity $\mathbf{v}$ we can formally make the substitution $\omega \rightarrow \mathrm{gv}$. Then both terms in parentheses contribute equally in Eq. (24), because one can set $\mathbf{g} \rightarrow-\mathbf{g}$ in the second term. This increases the resonant term of $v(\mathbf{v})$ for Bragg-Cherenkov scattering by a factor of 2 as compared to the ac resonant term, and it agrees accurately with the result found for the nonlinear conductivity [21] when $\alpha_{g}(T)<<1$. The one-phonon origin of the result given by Eq. (22) is very important for the strong-coupling treatment.

\section{Strong-coupling regime}

The WS DSF $S(\mathbf{q}, t)$ can be formally evaluated in terms of the new coupled phonon-ripplon modes. In the regime $\omega_{f}>>\omega_{g_{1}}$ the fast modes $\Omega_{p, k}^{(f)}$ represent electron oscillations in the field of steady dimples and $\left|\mathbf{E}_{p, k}^{(f)}\right|^{2} \simeq\left|\mathbf{E}_{p, k}\right|^{2}=1$ (here $\mathbf{E}_{p, k}^{(f)}$ is the polarization vector of the fast modes) [22]. Because the spectrum of the fast mode $\Omega_{t, k}^{(f)}$ has a quite high limiting frequency $\omega_{f}$, one can use the conventional phonon expansion to evaluate the contribution of this mode. This procedure was performed in Ref. 15 for high signal frequencies $\omega \sim \omega_{f}$. The important point is that at low frequencies $\omega \sim \omega_{g}$ under the strong-coupling-condition $\omega_{f}>>\omega_{g_{1}}$ there are no one-phonon terms involving fast phonons because of the energy conserving delta function, and the slow modes must be taken into account in order to find the contribution due to one-phonon processes.

For slow modes the spectrum of the transverse phonons can be found in an analytical form [2]

$$
\Omega_{t, k}^{(s)} \simeq \frac{\omega_{g_{1}} c_{t} k}{\sqrt{\omega_{f}^{2}+c_{t}^{2} k^{2}}}
$$


which is valid near the melting point, where coupling with ripplons of $q \simeq g_{1}$ dominates. At lower temperatures this equation can be used as a simple analytical interpolation. In this approximation the fact that the polarization vector $\mathbf{E}_{t, k}^{(s)}$ of the slow mode decreases rapidly when $k>\omega_{f} / c_{t}$ and this mode transforms into the pure ripplon mode with $\mathbf{q}=\mathbf{g}_{1} \pm \mathbf{k}$ must be taken into account [22]:

$$
\mathbf{E}_{t, k}^{(s)} \simeq \frac{\omega_{g_{1}}^{2}-\left(\Omega_{t, k}^{(s)}\right)^{2}}{\omega_{f} \omega_{g_{1}}} \mathbf{E}_{t, k} \equiv M_{t, k}^{(s)} \mathbf{E}_{t, k},
$$

where $\mathbf{E}_{t, k}$ is defined for a flat surface. Combining Eqs. (26) and (27) one can see that $M_{t, k}^{(s)} \propto k^{-2}$ for $k \gg \omega_{f} / c_{t}$. For small $k \ll \omega_{f} / c_{t}$ the quantity $M_{t, k}^{(s)} \simeq \omega_{g_{1}} / \omega_{f}<1$ reflects the mass increase due to surface dimples.

Substituting Eqs. (26) and (27) into the expression for $S^{(1-\mathrm{ph})}(\mathbf{q}, \omega)$ given in Eq. (14) we note that in one-phonon processes the phonon wavenumber

$$
k=k_{t}^{*} \equiv \frac{\omega_{f} \omega}{c_{t} \sqrt{\omega_{g_{1}}^{2}-\omega^{2}}}
$$

is even larger than that of the weak-coupling approach, where $k=k_{t} \equiv \omega / c_{t}$. Remarkably, the increase of the wavenumber $k$ and $1 /\left|\partial \Omega_{t, k}^{(s)} / \partial k\right|$ is exactly compensated by a decrease in $\left|\mathbf{E}_{p, k}^{(f)}\right|^{2}=\left(M_{t, k}^{(s)}\right)^{2}$. Therefore for $\mathbf{g}=\mathbf{g}_{1}$ we reproduce the one-phonon contribution to the electron DSF found in the weak-coupling treatment [Eq. (23)], except that energy conservation $\omega \pm \Omega_{t, k}^{(s)}=0$ restricts the possible values of the frequency argument because $\Omega_{t, k}^{(\mathrm{s})}<\omega_{g_{1}}$ :

$$
\begin{gathered}
S^{(1-\mathrm{ph})}(\mathbf{q}, \omega) \simeq \\
\simeq \frac{\pi \alpha_{q}}{|\omega|} \mathrm{e}^{-q^{2}\left\langle u_{f}^{2}\right\rangle / 2} \theta\left(\omega_{g_{1}}-|\omega|\right) N_{e} \sum_{|\mathbf{g}|=g_{1}} \delta_{\mathbf{q}, \mathbf{g}},
\end{gathered}
$$

where the reciprocal lattice vectors involved have the smallest absolute value $g_{1}$. An important consequence of the restriction $|\omega|<\omega_{g_{1}}$ is that the ripplon-absorption term of the effective collision frequency, containing $\left|\omega+\omega_{g}\right|^{-1}$, is zero in the strong-coupling treatment.

To understand the behavior of the electron DSF in the strong-coupling regime for $\alpha_{q}(T) \sim 1$, consider the contribution of the slow modes only $h_{q}^{(s)}(1, t)$. Using the actual forms for the slow phonon spectrum $\Omega_{t, k}^{(s)}$ and the polarization factor $M_{t, k}^{(s)}$ given above we obtain

$$
\begin{aligned}
& h_{q}^{(s)}(\mathrm{l}, t) \simeq \alpha_{q}(T) \int_{0}^{2 \pi} \frac{d \varphi}{2 \pi} \int_{0}^{\infty} \frac{d x}{x} \frac{\omega_{f}^{2}}{\omega_{f}^{2}+x^{2}} \times \\
& \times\left[1-\cos \left(\frac{x R_{1}}{c_{t}} \cos \varphi-\frac{x \omega_{g_{1}} t}{\sqrt{\omega_{f}^{2}+x^{2}}}\right)\right] .
\end{aligned}
$$

As compared to the weak-coupling theory, here the upper bound for the logarithmically large term is $\omega_{f}$ instead of $\omega_{m}$. Therefore, the strong-coupling theory approximation for $h_{q}^{(s)}(1, t)$, similar to Eq. (18), can be written as

$$
h_{q}^{(s)}(\mathbf{1}, t) \approx \alpha_{q}(T) \ln \left(\omega_{g_{1}} \sqrt{t^{2}+b R_{\mathbf{1}}^{2} /\left(c_{t}^{*}\right)^{2}}\right) .
$$

The complete function $h_{q}(1, t)$ also includes the contribution due to the fast modes $h_{q}^{(f)}(1, t)$. At low temperatures the latter can be approximated as $h_{q}^{(f)}(1, t) \simeq q^{2}\left\langle u_{f}^{2}\right\rangle / 2$. Thus, employing the approximation Eq. (18), in the strong-coupling regime we must make the substitutions $\omega_{m} \rightarrow \omega_{g_{1}}$ and $c_{t} \rightarrow c_{t}^{*}=$ $=c_{t} \omega_{g_{1}} / \omega_{f}$.

The analysis given above indicates that strong phonon-ripplon coupling does not change much the electron DSF for small values of the frequency argument $|\omega|<\omega_{g_{1}}$, if $\alpha_{q}(T)<<1$. As a result, the onephonon term in the effective collision frequency responsible for the unusual resonance shape of $v(\omega)$ at $\omega \sim \omega_{g_{1}}$ appears to be the same as in the weak-coupling theory. The important question is whether this form of the DSF can be used in Eqs. (4) and (8) for the conductivity relaxation kernel $M(\omega)$. The electron DSF appearing in these equations originates from the total interaction Hamiltonian given in Eq. (3). In the strong-coupling theory the interaction term proportional to $\xi_{\mathbf{q}} i \mathbf{q u} \mathbf{u}_{s, 1}$ is included in the Hamiltonian of the slow phonon modes (see Refs. 2, 22) which is then transformed to the canonical form of independent oscillators by a linear transformation of the normal coordinates. Therefore, if we consider scattering events in terms of the new (reconstructed) phonon modes, the term $\xi_{\mathbf{q}} i \mathbf{q u} \mathbf{u}_{s, \mathbf{l}}$ should be excluded from the scattering Hamiltonian.

It is clear that the rest of the interaction Hamiltonian in Eq. (3) cannot result in one-phonon scattering events involving slow phonons. Therefore, in the strong coupling regime it is reasonable to exclude the one-phonon terms $S^{(1-\mathrm{ph})}(\mathbf{q}, \omega)$ and $v^{(1-\mathrm{ph})}(\omega)$ from the conductivity relaxation kernel. This concerns Eq. (22) also, if we agree that it represents the conventional one-phonon term affected by long-wavelength fluctuations with $k<k_{t}$. The rest of the interaction Hamiltonian can result only in multi-phonon 
terms of the electron DSF which we are going to discuss below.

The electron-ripplon coupling $V_{q}$ entering the effective collision frequency is an increasing function of $q$ or a constant proportional to the holding electric field $E_{\perp}\left(V_{q} \simeq e E_{\perp}\right)$. This means that the main contribution to the sum $\sum_{q}$ of Eq. (10) is due to large wavenumbers $q$. In this case, the multi-phonon terms can be taken into account by a short-time approximation expanding $h_{q}$ in powers of $t$ near the minimum $(t=0)$. This approximation for $h_{q}(\mathbf{l}, t)$ and $S(\mathbf{q}, \omega)$ favors the contribution due to the fast modes $\Omega_{p, k}^{(f)}$ and $1=0$, which can be written in the following form well-known in the theory of thermal neutron scattering by crystals:

$$
S(q, \omega)=\hbar \sqrt{\frac{\pi}{\varepsilon_{q} K_{e}}} \exp \left[-\frac{\left(\varepsilon_{q}-\hbar \omega\right)^{2}}{4 \varepsilon_{q} K_{e}}\right],
$$

where $K_{e}$ is the mean kinetic energy per electron

$$
K_{e}=\left(2 N_{e}\right)^{-1} \sum_{p, \mathbf{k}} \hbar \Omega_{p, k}\left(n_{p, k}+1 / 2\right) .
$$

It is clear that at low temperatures the main contribution to $K_{e}$ is due to large wavenumbers.

In the Debye approximation

$$
\begin{aligned}
& K_{e}=T\left(\frac{2 T}{\hbar c_{t} k_{m}}\right)^{2} \int_{0}^{\hbar c_{t} k_{m} / 2 T} x^{2} \operatorname{coth}(x) d x+ \\
& +2 T\left(\frac{2 T}{\hbar \Omega_{l, k_{m}}}\right)^{4} \int_{0}^{\hbar \Omega_{l, k_{m}} / 2 T} x^{4} \operatorname{coth}(x) d x,
\end{aligned}
$$

where $k_{m}=\sqrt{4 \pi n_{s}}$. For $T>>\hbar c_{t} k_{m}$ this equation yields $K_{e} \rightarrow T$, but for lower $T$ the mean kinetic energy of electrons in the WS state is larger than $T$ because of the zero-point term. For a nondegenerate electron gas the typical wavenumbers $q \sim \sqrt{8 m_{e} T} / \hbar$, which make the main contribution to the effective collision frequency, decrease strongly with cooling. If the characteristic wavenumbers $q$ behaved in this manner in the WS state, the approximation Eq. (32) would fail rapidly as the temperature decreases. The important point is that for the WS $q_{e}=\sqrt{8 m_{e} K_{e}} / \hbar$ remains large because of the zero-point vibrations as shown in Fig. 2 [at $T>0.1 \mathrm{~K}$ it is substantially smaller than the wavenumbers of thermal ripplons $q_{T} \sim(T / \hbar)^{2 / 3}(\rho / \alpha)^{1 / 3}$ ]. This makes Eq. (32) a rea-

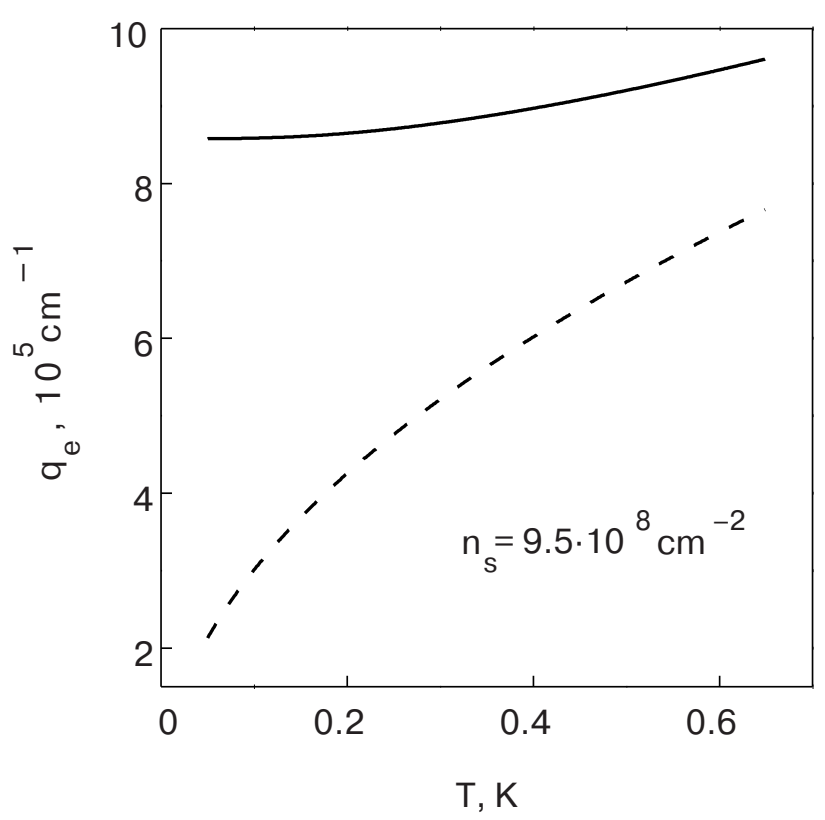

Fig. 2. Temperature dependence of the characteristic wavenumber $q_{e}$ of the electron dynamical structure factor for the WS (solid line) and gas (dashed line) states.

sonable approximation even at quite low temperatures.

The electron-ripplon coupling $V_{q}$ consists of the polarization term and the holding field term $e E_{\perp}$ [12]. Therefore the effective collision frequency, which follows from the short-time approximation of Eq. (32), can be written as

$$
v_{W S}=\frac{\left(e E_{\perp}\right)^{2}}{4 \alpha \hbar} \frac{2}{\sqrt{\pi}} \int_{0}^{\infty} U^{2}(x) \mathrm{e}^{-x^{2}} d x,
$$

where

$$
\begin{gathered}
U(x)=1+\frac{\Lambda q_{e}^{2}}{2 e E_{\perp}} x^{2} P\left(\frac{q_{e}^{2} x^{2}}{4 \gamma^{2}}\right), \\
\Lambda=\frac{\mathrm{e}^{2}(\epsilon-1)}{4(\epsilon+1)},
\end{gathered}
$$

$q_{e}=\sqrt{8 m_{e} K_{e}} / \hbar, \epsilon$ is the dielectric constant of liquid helium, and $P(y)$ is the electron-ripplon coupling function for the polarization term [at low temperatures $P(y) \simeq 0.5 \ln (4 / y)-1]$. In the limit of strong holding fields $V_{q} \simeq e E_{\perp}(U \simeq 1)$ the effective collision frequency $v_{W S}$ is, remarkably, independent of $K_{e}$ and is identical to that found for a nondegenerate electron gas with $v_{e e}>>v$. The high values of $q_{e}$ found in the WS state affect only the polarization term of Eq. (35). In particular, this increases the part of $v_{W S}$ that is linear in $E_{\perp}$. The ratio of $v_{W S}$ to the result found for the gas state $v_{\text {gas }}$ is shown in Fig. 3 


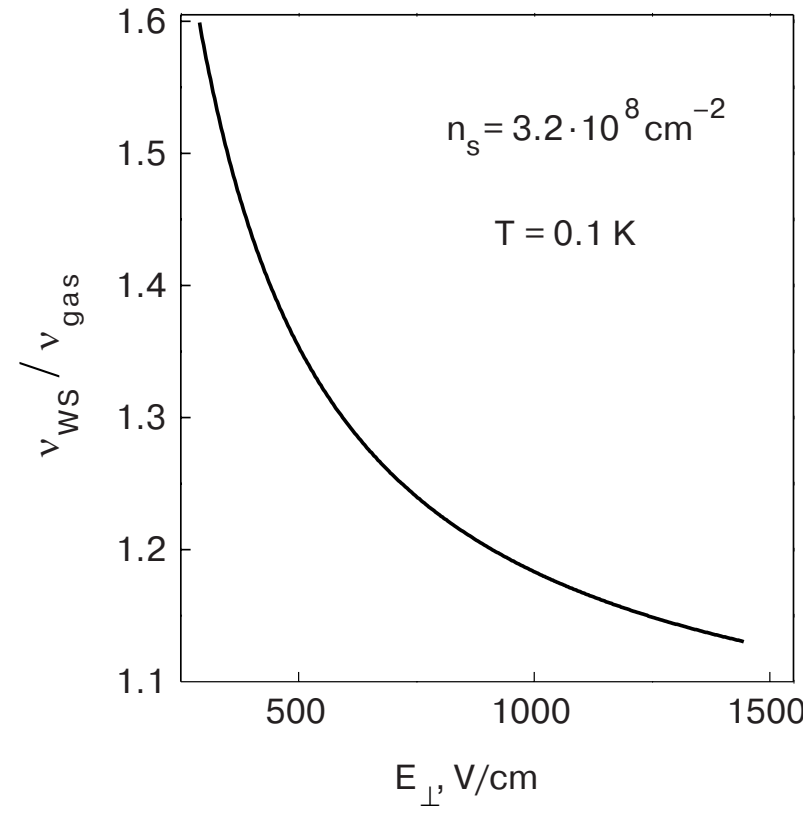

Fig. 3. The holding field dependence of the effective collision frequency of the WS normalized to the collision frequency of a nondegenerate electron gas.

as a function of the holding electric field $E_{\perp}$. This indicates that the effective collision frequency of the WS given in Eq. (34) is relatively larger than $v_{\text {gas }}$ for weaker holding fields $E_{\perp}$.

The magnitude of the multi-phonon terms can be determined by analyzing the two-phonon terms in the phonon expansion of the WS DSF. Consider, first, the contribution due to the fast modes whose frequencies are restricted by the condition $\Omega_{p, k}^{(f)}>\omega_{f}>>\omega_{g}$. Then energy conservation restricts the analysis to phonon scattering processes only, where the WS phonon frequencies enter the $\delta$-functions with opposite signs. In the temperature range $T>\hbar \omega_{f}$ direct evaluation yields

$$
S^{(2-\mathrm{ph})}(\mathbf{q}, \omega) \simeq \frac{\pi \alpha_{q}^{2}(T)}{2 \omega_{f}} \mathrm{e}^{-q^{2}\left\langle u_{f}^{2}\right\rangle / 2} N_{e} \sum_{\mathbf{g}} \delta_{\mathbf{q}, \mathbf{g}} .
$$

The respective contribution to the electron collision frequency can be written in the form

$$
v^{(2-\mathrm{ph})}=\frac{\pi n_{s}}{8 \alpha m_{e} \omega_{f}} \sum_{\mathrm{g}} \alpha_{g}^{2}(T) V_{g}^{2} \mathrm{e}^{-g^{2}\left\langle u_{f}^{2}\right\rangle / 2} .
$$

The temperature dependence of this term is determined by the interplay of two parameters: $\alpha_{g}(T) \propto T$ and $\left\langle u_{f}^{2}(T)\right\rangle$. As $\left\langle u_{f}^{2}(T)\right\rangle \rightarrow\left\langle u_{f}^{2}(0)\right\rangle$ we obviously have $v^{(2-\mathrm{ph})} \propto T^{2}$.

For slow modes $\left[\Omega_{p, k}^{(s)}<\omega_{g_{1}}\right]$ the situation is more difficult. Formal evaluation taking into account the polarization factors $M_{t, k}^{(s)}$ gives

$$
S_{\text {slow }}^{(2-\mathrm{ph})}(\mathbf{q}, \omega) \simeq \frac{\pi \alpha_{q}^{2}}{2|\omega|} \mathrm{e}^{-q^{2}\left\langle u_{f}^{2}\right\rangle / 2} J(\omega) N_{e} \sum_{|\mathbf{g}|=g_{1}} \delta_{\mathbf{q}, \mathbf{g}},
$$

where

$$
J(\omega)=\int_{y_{0}}^{y_{m}} \frac{d y}{y} \frac{|\omega| \theta\left(\omega_{g_{1}}-|\omega|\right)}{\left(1+y^{2}\right)\left(|\omega|+\omega_{g_{1}} y / \sqrt{1+y^{2}}\right)},
$$

$y_{m}=\omega / \sqrt{\omega_{g_{1}}^{2}-\omega^{2}}$, the lower limit $y_{0}=c k_{0} / \omega_{f}$, and $k_{0}$ is the smallest wavenumber of the finite electron system $\left(k_{0} \propto 1 / L\right)$. The integral $J(\omega)$ depends on the linear size $L$ of the system logarithmically as $J(\omega) \approx \ln \left(y_{m} / y_{0}\right)$, which indicates that the conventional phonon expansion of the WS DSF for the slow modes is asymptotic and the high-order terms cannot be used carelessly, especially if the parameter $\alpha_{g}$ is not small enough. Generally, Eq. (38) is similar to the result found for the fast modes [Eq. (36)]. The important point is that in the sum $\sum_{\mathrm{g}}$ the reciprocal lattice vectors are now restricted to ${ }^{\mathbf{g}}|\mathbf{g}|=g_{1}$ because the phonon frequencies are limited by $\omega_{g_{1}}$. This also indicates that the ripplon emission term in the effective collision frequency is zero because the argument $\omega+\omega_{g}$ of the DSF does not satisfy the requirement of Eq. (39).

There are also multi-ripplon scattering processes in which the ripplon wavenumbers $q$ are less restricted by the electron wavenumbers. For $q>>1 / \sqrt{\left\langle u_{f}^{2}\right\rangle}$ their contribution can be estimated by the geometrical optics approximation. The interaction changes the energy and velocity of ripplons in the electron localization area. The respective correction to the effective collision frequency of electrons is found as [23]

$$
\Delta v \approx \frac{\hbar^{3} \gamma^{4} \rho T^{2}}{4 \pi \alpha^{3} m^{3}\left(\left\langle u_{f}^{2}\right\rangle\right)^{3 / 2}} .
$$

This correction has a relatively weaker dependence on the holding electric field $\left(\Delta v \propto \gamma^{4} \propto E_{\perp}^{4 / 3}\right)$. Usually, it is much smaller than the result given by the short-time approximation Eq. (34).

\section{Results and discussion}

An important consequence of the weak-coupling result [14] given by Eqs. (20) and (22) is that the appearance of the electron-ripplon resonances generally 
does not coincide with the WS phase transition. Let us define the characteristic temperatures $T_{n}^{*}$ (here $n=1,2,3, \ldots)$ as the solutions of the equation $\alpha_{g_{n}}\left(T_{n}^{*}\right)=1$. Recalling the melting temperature of the 2D electron crystal $T_{m}=n_{s} a^{2} m_{e} c_{t}^{2} / 4 \pi$ given by the dislocation-melting theory [24] and the definition of $\alpha_{g}(T)$, we can see that for the triangular electron lattice the series $T_{n}^{*}$ is $T_{1}^{*}=3 T_{m}, T_{2}^{*}=T_{m}$, and $T_{3}^{*}=3 T_{m} / 4<T_{m}$. Therefore, near the WS phase transition the only resonance at $\omega \simeq \omega_{g_{1}}$ can exist in the presence of long-wavelength fluctuations. Still, at this resonant frequency there are no electron-ripplon resonances under the usual experimental conditions (they are shifted into the range $\omega<\omega_{g_{1}}$ ). The other two resonances $\omega \simeq \omega_{g_{2}}$ and $\omega=\omega_{g_{3}}$ cannot exist at $T \simeq T_{m}$ in the weak-coupling theory because the exponent $1-\alpha_{g}(T)$ appearing in the resonant conductivity term $\sigma \propto 1 /\left|\omega-\omega_{g}\right|^{1-\alpha_{g}}$ is zero or negative.

According to the relation between $T_{3}{ }^{*}$ and $T_{m}$ written above, as temperature decreases the weak-coupling theory gives a $25 \%$ delay in the appearance of the electron resonances with $\omega \simeq \omega_{g_{3}}$ as compared to the resonance with $\omega \simeq \omega_{g_{2}}$ and the WS phase transition. For example, the resonance $\mathbf{Z}$ of the experiment of Grimes and Adams [3] should not be observed before $T=(3 / 4) \cdot 0.45 \mathrm{~K} \simeq 0.345 \mathrm{~K}$. Still, it is clearly seen already at a substantially higher temperature $T=$ $=0.42 \mathrm{~K}$. A similar conclusion follows from a detailed study of the electron-ripplon resonances reported by Deville [7]. He observed the high-order resonances up to $\omega=\omega_{g_{13}}$. The high-order resonances indeed appear successively at progressively lower temperatures below $T_{m}$. It was reported that at $T \simeq T_{m} / 5$ modes up to $n=13$ can be detected for typical density $n \simeq$ $\simeq 2 \cdot 10^{8} \mathrm{~cm}^{-2}$. The characteristic temperature $T_{n}^{*}$, below which the resonance can be observed according to the weak-coupling theory under these conditions, is substantially lower than $T: T_{13}=3 T_{m} / 28 \simeq 0.1 T_{m}$. Thus both experiments show that the weak-coupling treatment of thermal fluctuations of the 2D WS overestimates the role of long-wavelength vibrations.

The discussion above pertains not only to the resonance structure of the effective collision rate $v(\omega)$. It is clear that the model DSF Eq. (20) cannot give the proper resonance structure of the real part of the conductivity relaxation kernel $\omega(\omega)=\operatorname{Re} M(\omega) \propto \omega /\left(\omega_{g}^{2}-\omega^{2}\right)$ which is responsible for the high-order coupled phonon-ripplon modes. Therefore the observation of the high-order resonances for $1-\alpha_{g}(T) \leq 0$ indicates that long-wavelength fluctuations of the 2D WS which are strongly coupled to media vibrations are less important for the conductivity relaxation kernel. An explanation of this surprising conclusion was given in the preceding section. It is based on the fact that the most important part of the electron-ripplon interaction proportional to $\xi_{\mathbf{q}} i \mathbf{q u} \mathbf{u}_{s, 1}$ is included in the Hamiltonian of the slow coupled phonon-ripplon modes. As a result, it cannot lead to any scattering of excitations of the slow modes. The long-wave fluctuations represent the excitations of slow coupled phonon-ripplon modes and therefore in the strong-coupling theory they affect the conductivity relaxation kernel to a much lesser extent. Then the appearance of the slow plasmon-ripplon resonance with $\omega<\omega_{g_{1}}$ obviously coincides with the WS phase transition, and the high-order resonances ( $\left.g_{n}>g_{1}\right)$ are not restricted by the condition $1-\alpha_{g}(T)>0$; this is important for experimental determination of the liquid-solid phase diagram.

For small wavenumbers $q$ the WS DSF $S(\mathbf{q}, \omega)$ which should be employed in the conductivity relaxation kernel of the strong-coupling theory has well-defined peaks $S(\mathbf{q}, \omega) \propto \delta(\omega) \sum_{\mathbf{g}} \delta_{\mathbf{q}, \mathbf{g}}$. This results in the characteristic form $\omega(\omega) \propto \omega /\left(\omega_{g}^{2}-\omega^{2}\right)$, which is important for the secular equation describing the slow coupled modes [Eq. (2)]. At low frequencies the main contribution to $w(\omega)$ is due to the smallest $g$, even if the DWF $\exp \left(-g^{2}\left\langle u_{f}^{2}\right\rangle / 2\right)$ is not small, because the corresponding terms of the sum over $\mathbf{g}$ are proportional to $1 / \omega_{g}^{2} \propto 1 / g^{3}$. At the same time the main contribution to the effective collision frequency $v(\omega)$ defined by Eq. (10) sometimes is due to large $q$, where the WS DSF can be described by the short-time approximation [Eq. (32)]. This follows from the general structure of Eqs. (10) and (32).

Another important result of the weak-coupling theory is the unusual line-shape with the non-Lorentzian tails: $\sigma \propto 1 /\left|\omega-\omega_{g}\right|^{1-\alpha_{g}}$. In contrast, the strong-coupling theory considered here results in the usual Lorentzian shape of the electron-ripplon resonances which is described by Eq. (1). Even if the sharp resonance structure of $v(\omega)$ becomes important, the line-shape of the resonance excitation of the slow plasmon-ripplon mode $\Omega_{l}^{(s)}\left(k_{0}\right)$, which is shifted substantially in the range $\omega<\omega_{g_{1}}$, is obviously close to a Lorentzian function. The presence of the nonresonance terms in the effective collision frequency $v(\omega)$ means that the tails of the high-order resonances $\left(g_{n}>g_{1}\right)$ are the usual Lorentzian functions determined by Eq. (1) as well.

Besides qualitative distinctions stated above, it is interesting to give a numerical comparison of the weak- and strong-coupling approaches for typical experimental conditions. Consider the effective collision 


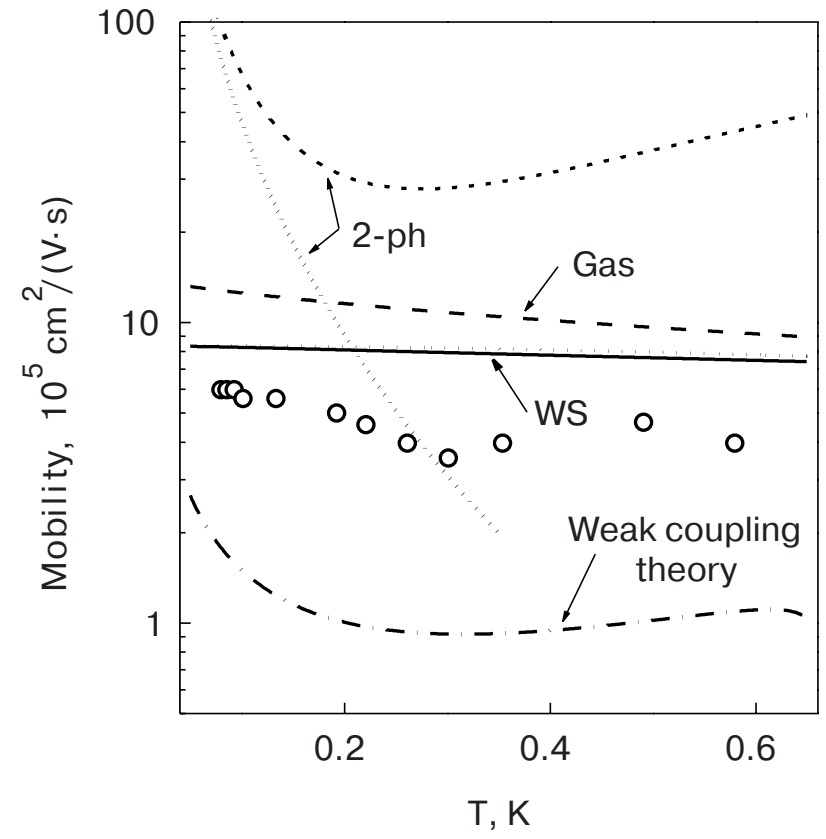

Fig. 4. Temperature dependence of the mobility of highly correlated electrons for different models of electron transport: the weak-coupling theory for the WS (dash-dotted line), mobility of a nondegenerate electron gas (dashed line), the WS mobility in the short-time approximation (dotted and solid lines; the latter includes the two-ripplon correction), the two-phonon term (short dashed and short dotted lines, as discussed in the text). The experimental data are taken from [9].

frequency $v$ of the WS and the corresponding mobility $\mu=e /\left(m_{e} v\right)$ which is related to the conductivity form of Eq. (1) at the resonance $\omega=\omega_{R}$. The results of different theoretical approximations and the experimental data of Ref. 9 are shown in Fig. 4. The weak-coupling theory result Eq. (22) (dash-dotted line) was calculated considering terms with $g<g_{4}$ only, because for $g \geq g_{4}$ the parameter $\alpha_{g}$ becomes larger than 1 and the approximation fails. Numerically it is approximately 6 times lower than the experimental data, which means that electron scattering by ripplons is overestimated in this approach. This would give a much broader resonance line-shape for the excitation of the slow plasmon-ripplon modes. The short time approximation for the WS DSF gives a substantially higher mobility (dotted line labelled «WS»). It is lower than the mobility evaluated for the gas state of the electron system (dashed line labelled «Gas»). Under the conditions considered the two-ripplon processes [Eq. (40)] have only a weak effect on the WS mobility (solid line).

The short-time approximation Eq. (34) describes the contribution of multi-phonon scattering events when the Debye - Waller function $2 q^{2} W(0,0)$ is large. The accuracy of this approximation can be estimated considering the contribution of two-phonon scattering events for two models of the WS DWF $\exp \left(-g^{2}\left\langle u^{2}\right\rangle / 2\right)$. If only zero-point vibrations are taken into account $\left(\left\langle u^{2}\right\rangle=\left\langle u_{0}^{2}\right\rangle\right)$ in Eq. (37) found for the fast modes, then the respective collision frequency increases rapidly with temperature and the WS mobility decreases as indicated by short-dot line labelled «2-ph». When thermal fluctuations are included $\left(\left\langle u^{2}\right\rangle=\left\langle u_{f}^{2}\right\rangle\right)$ the mobility curve (short-dash line labelled «2-ph») deviate strongly at $T>0.2 \mathrm{~K}$ and the effective collision frequency induced by two-phonon processes is drastically decreased. This means that in this temperature range $2 q^{2} W(0,0)$ is large and the short-time approximations Eqs. (32) and (34) are reasonable for the description of the WS conductivity.

The experimental data shown in Fig. 4 do not contradict the short-time approximation for the DSF. Still they indicate lower mobility values than that shown by the solid curve. The numerical difference may be due to the Debye approximation used to evaluate $K_{e}$ and $\left\langle u_{0}^{2}\right\rangle$. The influence of other mechanisms of momentum relaxation, which were disregarded in this work, cannot be ruled out. For example, it is well known that for the usual driving voltages the WS conductivity shows a remarkable nonlinear behavior $[10,11]$, which is not entirely understood.

In conclusion, we found that the quantum transport framework based on the memory function formalism is very fruitful for analyzing the WS conductivity in the low-frequency range, where it exhibits the resonance anomalies due to the interaction with media vibrations. The analytical properties of the memory function and its relationship with the electron dynamical structure factor makes it possible to coordinate the approximations used to describe the real part of the relaxation kernel, determining the positions of the resonance frequencies, and the effective collision frequency responsible for the broadening and line-shape of the resonances. For example, the model used to describe the secular equation for the coupled WS phonon-ripplon modes imposes strict consistency requirements on the approximations that can be used to obtain the effective collision frequency. The conductivity analysis given here for the strong-coupling regime eliminates the discrepancies between experiment and the weak-coupling approximation which concerns the line-shape and broadening of the conductivity resonances.

1. Yu.P. Monarkha and V.B. Shikin, Zh. Eksp. Teor. Phys. 68, 1423 (1975) [Sov. Phys. JETP 68, 710 (1975)].

2. D.S. Fisher, B.I. Halperin, and P.M. Platzman, Phys. Rev. Lett. 42, 798 (1979). 
3. C.C. Grimes and G. Adams, Phys. Rev. Lett. 42, 795 (1979).

4. A. G. Eguiluz, A. A. Maradudin and R. J. Elliott, Phys. Rev. B24, 197 (1981).

5. H. Namaizawa, Solid State Commun. 34, 607 (1980).

6. Yu.P. Monarkha and V.B. Shikin, Fiz. Nizk. Temp. 9, 913 (1983) [Sov. J. Low Temp. Phys. 9, 471 (1983)]

7. G. Deville, J. Low Temp. Phys. 72, 135 (1988).

8. V.E. Sivokon', V.V. Dotsenko, Yu.Z. Kovdrya, and V.N. Grigor'ev, Fiz. Nizk. Temp. 22, 1107 (1996) [Low Temp. Phys. 22, 845 (1996)].

9. V.V. Dotsenko, V.E. Sivokon, Yu.Z. Kovdrya, V.N. Grigor'ev, Physica B249, 648 (1998).

10. K. Shirahama and K. Kono, Phys. Rev. Lett. 74, 781 (1995).

11. A. Kristensen, K. Djerfi, P. Fozooni, M.J. Lea, P.J. Richardson, A. Santrich-Badal, A. Blackburn, and R.W. van der Heijden, Phys. Rev. Lett. 77, 1350 (1996).

12. Yu.P. Monarkha, E. Teske, and P. Wyder, Phys. Rep. 370, Issue 1 (2002).
13. O. Kirichek, M. Saitoh, K. Kono, and F.I.B. Williams, Phys. Rev. Lett. 86, 4064 (2001).

14. M.I. Dykman, Fiz. Nizk. Temp. 10, 453 (1984) [Sov. J. Low Temp. Phys. 10, 233 (1984)].

15. Yu.P. Monarkha and K. Kono, J. Phys. Soc. Jpn. 70, 1617 (2001).

16. W. Götze and P. Wölfle, Phys. Rev. B6, 1226 (1972).

17. Yu.P. Monarkha, Fiz. Nizk. Temp. 6, 685 (1980) [Sov. J. Low Temp. Phys. 6, 331 (1980)].

18. Yu.P. Monarkha, Fiz. Nizk. Temp. 7, 692 (1981) [Sov. J. Low Temp. Phys. 7, 338 (1981)].

19. B. Jancovici, Phys. Rev. Lett. 19, 20 (1967).

20. Y. Imry and L. Gunther, Phys. Rev. B3, 3939 (1971).

21. M.I. Dykman and Yu.G. Rubo, Phys. Rev. Lett. 78 4813 (1997)

22. Yu.P. Monarkha and S.S. Sokolov, Fiz. Nizk. Temp. 8, 350 (1982) [Sov. J. Low Temp. Phys. 8, 173 (1982)].

23. Yu.P. Monarkha, Fiz. Nizk. Temp. 19, 235 (1993) [Sov. J. Low Temp. Phys. 19, 163 (1993)].

24. D.J. Thouless, J. Phys. C11, L189 (1978) 